\title{
sciendo
}

\section{Analytic Method for Decision Support of Blockchain Technology Supplier Selection in Industry 4.0 Era}

doi:10.2478/mape-2020-0026

Date of submission to the Editor: 03/2020

Date of acceptance by the Editor: 06/2020

MAPE 2020, volume 3, issue 1, pp. 296-307

\section{Witold Torbacki}

ORCID ID: 0000-0001-9924-0431

Maritime University of Szczecin, Poland

\section{INTRODUCTION}

Blockchain methods are not mass solutions, in most cases, they are closely matched to the needs. The possibility of extending the standard method very often determines the success of the implementation.

Managing staff in manufacturing companies, choosing a new IT solution, may not pay attention to the important elements determining the success of the implementation.

The article is a kind of compendium of information and a guide that presents the most important criteria for blockchain integrator selection and facilitates the selection of modern blockchain technology in manufacturing companies that would like to secure the data transfer in Industry 4.0 solutions. Parameters are analyzed using the DEMATEL method (Du and Zhou, 2019). The significance of individual parameters and their interrelationships are established.

\section{INDUSTRY 4.0 AND BLOCKCHAIN}

The term Industry 4.0 is often used to describe the changes related to the fourth industrial revolution. The concept of Industry 4.0 involves the participation of modern technologies that change the modern industry. These include, among others the Internet of Things, the Internet of Services, intelligent factories and production, artificial intelligence, robotics, digital transformation, augmented reality, man-machine interfaces, as well as cyber-physical systems (Bai et al., 2020, Corallo et al., 2020). An essential element combining these solutions within Industry 4.0 is the widespread use of the data transfer in operational work. The graphic WiFi symbol means data logging using cyber-physical systems and the blockchain symbol means the protected transmission of collected data (Fig. 1).

In the era of Industry 4.0 (Liu et al., 2020, Lee et al., 2019, Aheleroff et al., 2020), the issue of secured data transfer between all participants in an order chain is of particular importance. This chain covers marketing and sales activities, orders from customers, orders for suppliers, production, distribution and service processes. Blockchain technology used to secure transferred electronic files 
ensures them so-called the durability of the record as well as the integrity of the content and the undeniable origin.

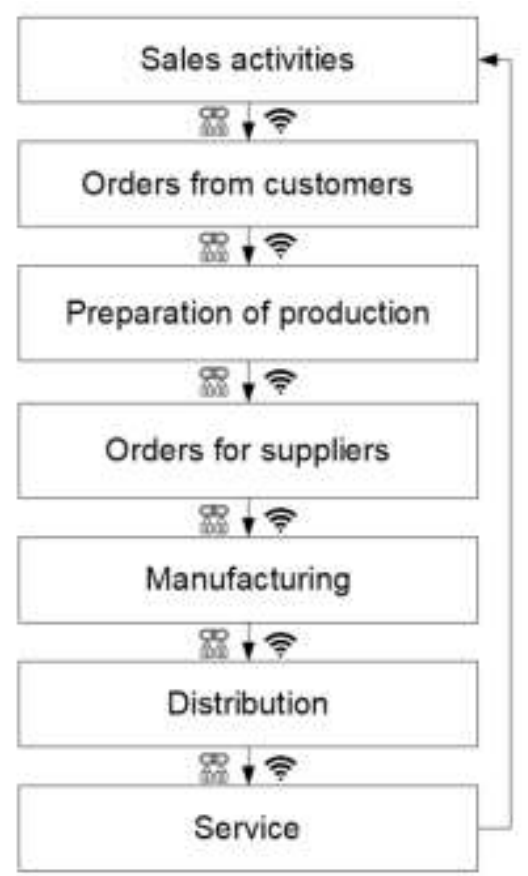

Fig. 1 The blockchain-secured processing of orders, production and distribution in the Industry 4.0 era

It is also characterized by comprehensive use of the Internet of things and cyberphysical systems for monitoring, optimization and initiation of processes. In the production cycle, this means that IT systems register and monitor the course of physical processes, which, in the feedback loop, affect the process of data processing in the systems (Reyna et al., 2018).

\section{BLOCKCHAIN}

Blockchain technology is gaining more and more attention in many companies. Blockchain is a block of chains that is a public record of all transactions in chronological order. This chain is common to all users of a given process. In practice, information blocks are connected in a chain, in which each subsequent element depends on the previous one. Blockchain provides each participant with the entire database of such a register. This creates a completely centralized register. This solution makes it impossible to modify data, delete or add false information.

Electronic documents, invoices, digitally secured and issued by companies can be an excellent illustration of the use of this technology. Four types of electronic document security are generally used in companies: a digital stamp, nonqualified and qualified signature, and blockchain.

The digital stamp is usually issued in companies for entire departments. In the example with an invoice, it means that the invoice issued by the company's sales 
department can be digitally stamped, without explicitly indicating the author as an individual.

A qualified digital signature requires a personal verification of the identity of the person at a designated identity confirmation point. A qualified signature has legal effects and is equivalent to a handwritten signature. A document with a qualified signature may play an evidentiary role in the court process. The above features of a qualified signature do not have an unqualified signature, which can be issued by individual companies and organizations for their own needs and recognized by their contractors.

Returning to the example with an invoice, if it were signed with one of the above three methods, i.e. a stamp or two types of signature, this would not prevent the possibility of its disappearance. In the case of an invoice signed with a blockchain, it cannot be lost without a trace.

Blockchain consists of a chain of related to each other documents. The main chain of invoices consists of the series of blocks from the genesis block to the current block. Of course, as with documents secured by signature, blockchain also explicitly verifies the issuer of the document.

Blockchain technology, in principle, excludes the existence of any central body implementing changes in the process covered by blockchain technology, as the current version of the database can be scattered around the world. What is more, blockchain is not based on a secret and patented algorithm, but a fully open-source code, which can be analyzed and checked by practically anyone who knows software development. However, the question arises whether this technology is advanced and secure enough to process and secure important and sensitive data.

The basic feature of blockchain is 'non-repudiation.' In practice, it means that once recorded information remains unchanged forever, and any previously entered record cannot be deleted or falsified. Messages about new events are sent simultaneously to all system participants.

Any new information in the blockchain registry must be encrypted using a key pair (a public and private key). This involves generating a secure cryptographic signature. The public key encrypts information, while the private key is used to authorize sent messages as well as to read encrypted content by the recipient. The security of the account depends primarily on whether the user can securely store the private key assigned to them. It is worth noting that "losing" such key causes irreversible, both for the user and all third party except for a potential "key finder," loss of control over the account.

This signature is unique for each data set because it is based on the information about the sender, content and time of the event. The information encrypted in this way must be sent in an appropriate format and be verifiable. Verification is simple because every user of a blockchain-based system can check the correctness of any change in the system at any time. This leads to full decentralization, which prevents registers from being controlled by individuals (system administrators, lawyers) or organizations (banks, financial institutions, 
countries). What is more, at the time of updating, the information is distributed, available for inspection and verification to every system participant. The information from the register of all users is public. Instead of the name of the holder, there is a unique identifier.

\section{BLOCKCHAIN - EXAMPLES OF USAGE}

Blockchain grew out of financial applications and the term is associated primarily with cryptocurrencies, including Bitcoin. However, at present, you cannot concentrate solely on the financial use of blockchain technology (Yadav and Singh, 2020, Frizzo-Barker et al., 2020). Currently, it is a revolutionary solution that strongly threatens the existing status quo in the economy. The growing importance of blockchain can redefine the concept of trust, which until now was based on the authority of the institution, and now it can be based on the strength of the cryptographic algorithm used in the system.

Blockchain is currently becoming a subject of increasing interest from large corporations and states. Some examples of applications are presented below.

Banking sector - uses blockchain to verify the authenticity of documents. This solution significantly reduces the costs associated with the use of paper because all customers who declare an electronic form of contact do not receive documents by mail. Thanks to the blockchain reference, customers can verify that the current table of bank fees or the loan agreement presented by the bank are the same as those originally signed and have not changed.

Cryptocurrencies - blockchain allows them to function without having a central banking authority, which eliminates all fees associated with making transactions. Health care sector - this technology can be used to store patients' medical records securely. Registers are coded and stored in blockchain with a private key, thanks to which they are available only to authorized persons.

Real estate sector - the process of registering property rights is burdensome, inefficient and costly and, at the same time, consumes a lot of time. It is also full of human errors, which poses great difficulty in tracking property ownership. The use of blockchain technology in this sector makes it easy to track document history.

Public elections (Wang et al., 2018) - blockchain helps eliminate fraud related to falsification of election votes. Each voice can be stored as a block in a blockchain, which makes manipulation difficult.

Transport sector (Koh et al., 2020, Helo et al., 2019) - suppliers can use the potential of blockchain by registering the origin of purchased materials. This allows, above all, to verify the authenticity of the products. Maersk, the biggest container transport company in the world, has created the blockchain TradeLens platform in collaboration with IBM. It is supported by a group of the largest industry players. It provides a secure source of information for all stakeholders involved in the supply chain. Currently, there are several hundred carriers, ports, terminal operators and forwarders on TradeLens. 


\section{INTEGRATOR OF BLOCKCHAIN SELECTION CRITERIA}

Reliable indicators of the quality assessment of the blockchain technology are necessary for the correct selection of the blockchain platform and provider. The future blockchain user should consciously choose an integrator guaranteeing a sufficiently high level of service and a type of a blockchain.

Below, a set of 12 parameters is proposed, which should be considered when choosing both a variety of a blockchain and an integrator during the implementation of this technology (Table 1). The correct order of the selection process should include issues with the selection of the implementation partner (perspective A in Table 1), a blockchain architecture (perspective B) and the implementation process (perspective $\mathrm{C}$ ).

Table 1 Parameters of the choice of Blockchain technology integrator

\begin{tabular}{|l|l|l|}
\hline Perspective & KPI & Description \\
\hline \multirow{4}{*}{$\begin{array}{l}\text { Blockchain implementation } \\
\text { integrator }(\mathrm{A})\end{array}$} & A1 & Technical capability \\
\cline { 2 - 3 } & A2 & References of the blockchain integrator \\
\cline { 2 - 3 } & A3 & Innovativeness \\
\cline { 2 - 3 } & A4 & Flexibility \\
\hline \multirow{5}{*}{ Blockchain architecture (B) } & B1 & Market maturity of the solution \\
\cline { 2 - 3 } & B2 & Hacker resistance \\
\cline { 2 - 3 } & B3 & Technological advancement \\
\cline { 2 - 3 } & B4 & Data Security procedures \\
\hline \multirow{4}{*}{ Implementation process (C) } & C1 & Service quality \\
\cline { 2 - 3 } & C2 & Cost of implementation \\
\cline { 2 - 3 } & C3 & Roadmap of software development \\
\cline { 2 - 3 } & C4 & Warranties \& Contract \\
\hline
\end{tabular}

Technical capability (A1) influences the whole implementation project, either positively or negatively. An integrator may not have both the capability of technical knowledge and sufficient resources.

References of the blockchain integrator (A2). It is worth obtaining a reference list of similar implementations, financial results and a list of employees with qualifications suitable for a blockchain implementation from the integrator.

Innovativeness (A3). This enables the solution buyer to receive the software that gives a technological market advantage.

Flexibility (A4). It is important to check whether the integrator can absorb from other sources and implement new technologies quickly and whether relevant procedures have been prepared in this regard.

Market maturity of the solution (B1). It should be verified whether the implemented version of blockchain technology has its market history or whether it is a completely new solution, usually having various gaps.

Hacker resistance (B2). Verifying the security of the new solution at an early stage of testing increases the chance of winning among mass customers because every slip, a gap in the system, a failure in the clash with hackers cause media hysteria. As a result, the innovative product does not withstand criticism and disappears from the market. 
Technological advancement (B3). The employed technologies should provide reliability and scalability of the solution. It is necessary to get an idea of how IT technologies have been used to create the software, what platforms the software is designed for, and on what devices it can be launched.

Data Security procedures (B4). In 2019, the CEO of the cryptocurrency management fund passed away, and with him, the credentials for access to his cryptocurrencies managed for about USD 200 million were lost. They are impossible to recover. This is not the fault of a blockchain. It is the company that did not implement appropriate procedures that would have prevented such a scenario.

Service quality (C1). It is one of the most essential criteria for implementation. Service includes a response to complaints, channels of a communication system and predictive maintenance.

Cost of implementation (C2). A project TCO should be set. It is important to check whether the agreed project costs include software updates and postimplementation care.

Roadmap of software development (C3). Software and IT platforms are constantly changing. It is worth taking care of the development directions of the software. This will allow identifying development requests on time.

Warranties \& Contract (C4). It is useful to sign a warranty contract under which the integrator defines a minimum, guaranteed and measurable level of services offered, the violation of which often results in the payment of compensation.

\section{DEMATEL METHOD}

DEMATEL technique (Gabus, 1972) is one of Multi-Criteria Decision Analysis (MCDA) method. It is used to model and assess the interrelations between the parameters. This useful method is used to analyse the complex relationships among coefficients in a complex system for making a strategic decision. In this article, DEMATEL method is used to identify a correlation between sets of twelve factors for blockchain integrator selection. Moreover, it facilitates the selection of modern blockchain technology in manufacturing companies that would like to secure the data transfer in Industry 4.0 solutions. DEMATEL method divides the interrelations between factors into a reason group and an effect group.

The DEMATEL consists of eight stages.

Stage 1. A list of $k$ parameters, which undergoes the analysis, is appointed.

Stage 2. A group of $n$ experts are established. Each of them indicates the direct influence between different factors, using a numeric scale from 4 (very high influence) to 0 (no influence). Then the values of mutual interactions within pairs of all the criteria are determined. It is assumed that each of the $k$ factors may directly influence another factor, but it cannot influence itself. As a result, $n$ partitive initial direct influence matrices $\boldsymbol{Z}_{m}$ were created by each $m$-th expert:

$$
\boldsymbol{Z}_{m}=\left[z_{i j}^{m}\right]_{k \times k}
$$

where: 
$z_{i j}^{m}$ represents the assessment provided by the $m$-th expert regarding the degree to which parameter $i$ influences parameter $j$. A set of partial matrices is made.

Stage 3. Aggregation of these matrices gives a direct influence matrix $\boldsymbol{Z}=$ $\left[z_{i j}\right]_{k \times k}$ which is received by:

$$
\boldsymbol{Z}=\frac{1}{n} \sum_{m=1}^{n}\left[z_{i j}^{m}\right], \quad i, j=1,2,3, \ldots k
$$

Stage 4. Normalized direct influence matrix $X$ is obtained by using equations (3)-(4).

$$
\boldsymbol{X}=\left[x_{i j}\right]_{k \times k}=\frac{Z}{s}
$$

where:

$$
s=\max \left(\max _{1 \leq i \leq k} \sum_{j=1}^{k} z_{i j}, \max _{1 \leq j \leq k} \sum_{i=1}^{k} z_{i j}\right)
$$

Stage 5. Matrix of total relations $\boldsymbol{T}=\left[t_{i j}\right]_{k \times k}$ is created by

$$
\boldsymbol{T}=\boldsymbol{X}(\boldsymbol{I}-\boldsymbol{X})^{-1}
$$

where:

$I$ is an identity matrix.

Stage 6. The vectors $\boldsymbol{C}$ and $\boldsymbol{R}$ representing the sum of the columns and the sum of the rows from the matrix $\boldsymbol{T}$ are created by

and

$$
\boldsymbol{C}=\left[c_{j}\right]_{1 \times k}=\left[\sum_{j=1}^{k} t_{i j}\right]_{1 \times k}^{T}
$$

where:

$$
\boldsymbol{R}=\left[r_{i}\right]_{k \times 1}=\left[\sum_{i=1}^{k} t_{i j}\right]_{k \times 1}
$$

$G$ is the $j$-th column sum in the matrix $\boldsymbol{T}$.

It reflects the effects that parameter $j$ is receiving from all the other ones. Likewise, $r_{i}$ is the $i$-th row sum in the matrix $\boldsymbol{T}$. It presents the sum of direct and indirect effects dispatching from parameter $i$ to the other ones.

Let $i=j$ and $i, j \in\{1,2, \ldots, k\}$, a relation indicator $r_{i}-c_{j}$ is derived. It is also called a net influence. The vertical relation axis vector $\boldsymbol{R}-\boldsymbol{C}$ reflects the net effect that the factor contributes to the complex system. Similarly, a position indicator $r_{i}+c_{j}$ is made. The horizontal position axis $\boldsymbol{R}+\boldsymbol{C}$ shows the total effect of each factor on the whole system and reflects the degree of the role that the coefficient plays in the analysed system. As for $\boldsymbol{R}-\boldsymbol{C}$, the value of $r_{i}-c_{j}>$ 0 stands for that criterion $i$ influences other criteria as well as the entire system. On the other hand, the value $r_{i}-c_{j}<0$ means that other criteria influence criterion $i$.

Stage 7. Based on $\boldsymbol{T}=\left[t_{i j}\right]_{k \times k}$ matrix, the influential relation diagram in the $\left(r_{i}+c_{j}, r_{i}-c_{j}\right)$ the layout can be plotted.

Stage 8. DEMATEL analysis determines the level of correlations among coefficients. The position of each criterion in the diagram gives the information about their importance or has a complex relationship with other criteria. 


\section{INTEGRATOR OF BLOCKCHAIN SELECTION CRITERIA - CASE STUDY}

To appoint the parameters shown in Table 1, eight expert interviews are executed. Two of the experts have deep knowledge in blockchain technology, the next three are experts in the area of smart manufacturing, especially in the Industry 4.0 context. The sixth is an expert in classic manufacturing. The last two are experts in both blockchain and Industry 4.0.

They establish three perspectives and the set of twelve criteria presented in Table 1 above. These parameters should be a guide in the process of choosing the integrator of blockchain technology used for securing the flow of electronic company transactions in Industry 4.0 processes.

The experts assess correlations between pairs of all factors by using a 5-grade scale (from 0 equals 'no influence' to 4 equals 'very high influence'). Finally, eight partitive initial direct influence matrices $\boldsymbol{Z}_{m}$ are received, based on the equation (1). Superposition of the matrices, under equation (2), gives direct influence matrix $\boldsymbol{Z}$ (Table 2).

Table 2 Direct influence matrix $Z$

\begin{tabular}{|c|l|l|l|c|l|l|l|l|l|l|l|l|}
\hline $\boldsymbol{Z}$ & \multicolumn{1}{|c|}{ A1 } & \multicolumn{1}{|c|}{ 2 } & A3 & A4 & B1 & B2 & B3 & B4 & C1 & C2 & C3 & C4 \\
\hline A1 & 0 & 1.625 & 1.875 & 2.25 & 2.375 & 2.375 & 1.5 & 2.375 & 1.375 & 1.625 & 1.25 & 2.75 \\
\hline A2 & 2.375 & 0 & 1.625 & 2.375 & 1.875 & 3.625 & 1.875 & 2.5 & 1.625 & 2.375 & 1.625 & 1.75 \\
\hline A3 & 1.625 & 1.75 & 0 & 3.625 & 2.75 & 3.875 & 3.5 & 3.25 & 1.875 & 1.5 & 1.875 & 3.25 \\
\hline A4 & 2 & 3.25 & 3.5 & 0 & 1.875 & 3.625 & 1.5 & 3.875 & 2.75 & 2.875 & 3.75 & 1.875 \\
\hline B1 & 1.375 & 3.75 & 1.625 & 2.375 & 0 & 2.5 & 1.625 & 2.5 & 3 & 2.375 & 2.75 & 2.125 \\
\hline B2 & 2.25 & 1.5 & 1.875 & 3.875 & 2.375 & 0 & 2.375 & 1.5 & 2.25 & 1.75 & 1.375 & 2 \\
\hline B3 & 1.5 & 2 & 2.375 & 1.5 & 1.875 & 1.75 & 0 & 2.375 & 2.75 & 1.875 & 2.25 & 1.5 \\
\hline B4 & 2.75 & 2.25 & 1.375 & 3.375 & 2.5 & 3.875 & 1.875 & 0 & 1.5 & 2.5 & 1.625 & 3.125 \\
\hline C1 & 1.25 & 2.75 & 2 & 3.125 & 1.625 & 2.375 & 1.5 & 3.25 & 0 & 2.375 & 1.875 & 3.5 \\
\hline C2 & 1.5 & 1.75 & 3.75 & 2.5 & 1.75 & 2.25 & 2.375 & 2.75 & 1.5 & 0 & 1.375 & 2 \\
\hline C3 & 1.625 & 3.375 & 1.875 & 2.375 & 1.875 & 3.375 & 3.5 & 2.125 & 1.875 & 3.5 & 0 & 2.75 \\
\hline C4 & 2.75 & 2.375 & 2.125 & 3 & 1.5 & 2.375 & 2.625 & 3.25 & 1.625 & 2.125 & 1.875 & 0 \\
\hline
\end{tabular}

Based on equation (3), normalized direct influence matrix $\boldsymbol{X}$ (Table 3 ) is determined.

Table 3 Normalized direct influence matrix $X$

\begin{tabular}{|c|l|l|c|c|c|c|c|c|c|c|c|c|}
\hline $\boldsymbol{X}$ & \multicolumn{1}{|c|}{ A1 } & \multicolumn{1}{|c|}{ A2 } & A3 & A4 & B1 & B2 & B3 & B4 & C1 & C2 & C3 & C4 \\
\hline A1 & 0 & 0.051 & 0.059 & 0.07 & 0.074 & 0.074 & 0.047 & 0.074 & 0.043 & 0.051 & 0.039 & 0.086 \\
\hline A2 & 0.074 & 0 & 0.051 & 0.074 & 0.059 & 0.113 & 0.059 & 0.078 & 0.051 & 0.074 & 0.051 & 0.055 \\
\hline A3 & 0.051 & 0.055 & 0 & 0.113 & 0.086 & 0.121 & 0.109 & 0.102 & 0.059 & 0.047 & 0.059 & 0.102 \\
\hline A4 & 0.063 & 0.102 & 0.109 & 0 & 0.059 & 0.113 & 0.047 & 0.121 & 0.086 & 0.09 & 0.117 & 0.059 \\
\hline B1 & 0.043 & 0.117 & 0.051 & 0.074 & 0 & 0.078 & 0.051 & 0.078 & 0.094 & 0.074 & 0.086 & 0.066 \\
\hline B2 & 0.07 & 0.047 & 0.059 & 0.121 & 0.074 & 0 & 0.074 & 0.047 & 0.07 & 0.055 & 0.043 & 0.063 \\
\hline B3 & 0.047 & 0.063 & 0.074 & 0.047 & 0.059 & 0.055 & 0 & 0.074 & 0.086 & 0.059 & 0.07 & 0.047 \\
\hline B4 & 0.086 & 0.07 & 0.043 & 0.105 & 0.078 & 0.121 & 0.059 & 0 & 0.047 & 0.078 & 0.051 & 0.098 \\
\hline C1 & 0.039 & 0.086 & 0.063 & 0.098 & 0.051 & 0.074 & 0.047 & 0.102 & 0 & 0.074 & 0.059 & 0.109 \\
\hline C2 & 0.047 & 0.055 & 0.117 & 0.078 & 0.055 & 0.07 & 0.074 & 0.086 & 0.047 & 0 & 0.43 & 0.063 \\
\hline C3 & 0.051 & 0.105 & 0.059 & 0.074 & 0.059 & 0.105 & 0.109 & 0.066 & 0.059 & 0.109 & 0 & 0.086 \\
\hline C4 & 0.086 & 0.074 & 0.066 & 0.094 & 0.047 & 0.074 & 0.082 & 0.102 & 0.051 & 0.066 & 0.059 & 0 \\
\hline
\end{tabular}

Following the equation (5), the matrix of total relations $\boldsymbol{T}$ (Table 4 ) is made. 
Table 4 The matrix of total relations $T$

\begin{tabular}{|r|c|l|c|l|c|c|c|c|c|c|c|c|}
\hline $\boldsymbol{T}$ & $\mathrm{A} 1$ & $\mathrm{~A} 2$ & $\mathrm{~A} 3$ & $\mathrm{~A} 4$ & $\mathrm{~B} 1$ & $\mathrm{~B} 2$ & $\mathrm{~B} 3$ & $\mathrm{~B} 4$ & $\mathrm{C} 1$ & $\mathrm{C} 2$ & $\mathrm{C} 3$ & C4 \\
\hline A1 & 0.193 & 0.28 & 0.269 & 0.335 & 0.27 & 0.35 & 0.259 & 0.329 & 0.24 & 0.268 & 0.233 & 0.312 \\
\hline A2 & 0.278 & 0.25 & 0.282 & 0.362 & 0.274 & 0.409 & 0.288 & 0.354 & 0.264 & 0.308 & 0.259 & 0.304 \\
\hline A3 & 0.304 & 0.359 & 0.284 & 0.46 & 0.345 & 0.482 & 0.384 & 0.438 & 0.321 & 0.337 & 0.316 & 0.4 \\
\hline A4 & 0.331 & 0.42 & 0.402 & 0.383 & 0.34 & 0.504 & 0.351 & 0.477 & 0.358 & 0.396 & 0.38 & 0.387 \\
\hline B1 & 0.272 & 0.385 & 0.305 & 0.392 & 0.239 & 0.411 & 0.305 & 0.385 & 0.324 & 0.335 & 0.313 & 0.341 \\
\hline B2 & 0.272 & 0.297 & 0.289 & 0.4 & 0.285 & 0.304 & 0.299 & 0.329 & 0.282 & 0.29 & 0.255 & 0.31 \\
\hline B3 & 0.239 & 0.294 & 0.286 & 0.319 & 0.259 & 0.338 & 0.218 & 0.334 & 0.281 & 0.28 & 0.263 & 0.283 \\
\hline B4 & 0.316 & 0.348 & 0.305 & 0.426 & 0.318 & 0.453 & 0.317 & 0.318 & 0.288 & 0.342 & 0.287 & 0.373 \\
\hline C1 & 0.269 & 0.355 & 0.315 & 0.412 & 0.287 & 0.406 & 0.301 & 0.405 & 0.235 & 0.333 & 0.288 & 0.377 \\
\hline C2 & 0.256 & 0.305 & 0.343 & 0.37 & 0.274 & 0.377 & 0.306 & 0.367 & 0.263 & 0.241 & 0.256 & 0.315 \\
\hline C3 & 0.294 & 0.391 & 0.331 & 0.412 & 0.311 & 0.455 & 0.375 & 0.395 & 0.309 & 0.382 & 0.248 & 0.375 \\
\hline C4 & 0.307 & 0.341 & 0.315 & 0.403 & 0.282 & 0.402 & 0.328 & 0.4 & 0.282 & 0.322 & 0.285 & 0.274 \\
\hline
\end{tabular}

Matrix of total relations $\boldsymbol{T}$ can be treated as a set of two submatrices. The first one is a submatrix $\boldsymbol{T}_{\boldsymbol{P}}$ based on the three perspectives given in Table 1, the second one is a submatrix $\boldsymbol{T}_{K}$ based on twelve factors. Table 5 presents submatrices $\boldsymbol{T}_{\boldsymbol{P}}$ and $\boldsymbol{T}_{\boldsymbol{K}}$ and respective indicators $r_{i}$ and $c_{i}$ stated by the equations (6-7) as well as the relation indicators $r_{i}-c_{i}$ and position indicators $r_{i}+c_{i}$.

Table 5 The $T_{\mathrm{p}}$ and $T_{\mathrm{K}}$ submatrices and related indicators

\begin{tabular}{|c|c|c|c|c|c|c|c|c|c|}
\hline$T_{p}$ & $r_{i}$ & $c_{i}$ & $r_{i}+c_{i}$ & $r_{i}-c_{i}$ & $T_{K}$ & $r_{i}$ & $c_{i}$ & $r_{i}+c_{i}$ & $r_{i}-c_{i}$ \\
\hline \multirow{4}{*}{ A } & \multirow{4}{*}{1.008} & \multirow{4}{*}{0.985} & \multirow{4}{*}{1.993} & \multirow{4}{*}{0.023} & A1 & 3.336 & 3.328 & 6.665 & 0.0008 \\
\hline & & & & & A2 & 3.632 & 4.024 & 7.656 & -0.393 \\
\hline & & & & & A3 & 4.431 & 3.728 & 8.158 & 0.703 \\
\hline & & & & & A4 & 4.73 & 4.675 & 9.404 & 0.055 \\
\hline \multirow{4}{*}{ B } & \multirow{4}{*}{0.944} & \multirow{4}{*}{1.04} & \multirow{4}{*}{1.984} & \multirow{4}{*}{-0.096} & B1 & 4.007 & 3.485 & 7.491 & 0.522 \\
\hline & & & & & B2 & 3.612 & 4.891 & 8.503 & -1.28 \\
\hline & & & & & B3 & 3.392 & 3.73 & 7.123 & -0.338 \\
\hline & & & & & B4 & 4.092 & 4.53 & 8.622 & -0.438 \\
\hline \multirow{4}{*}{ C } & \multirow{4}{*}{0.992} & \multirow{4}{*}{0.92} & \multirow{4}{*}{1.912} & \multirow{4}{*}{0.072} & C1 & 3.982 & 3.446 & 7.428 & 0.536 \\
\hline & & & & & C2 & 3.671 & 3.833 & 7.505 & -0.162 \\
\hline & & & & & C3 & 4.278 & 3.382 & 7.66 & 0.896 \\
\hline & & & & & C4 & 3.941 & 4.052 & 7.993 & -0.111 \\
\hline
\end{tabular}

Regarding the submatrix $\boldsymbol{T}_{\mathbf{p}}$ the influential relation diagram can be plotted (Figure 2) for three perspectives from Table 1.

In Fig. 2, perspective A - Blockchain implementation integrator has the highest value of position indicator $r_{i}+c_{i}$. It is thought that it gives a possibility to reflect the parameter priority among the others. This perspective is the most strongly interconnected with the other perspectives as well as it is the most important out of the presented three perspectives. Perspective $\mathrm{C}-$ Implementation process shows the lowest level of this indicator. The relation indicator $r_{i}-c_{i}$ reflects the influence of the analysed parameter on the other factors. In Fig. 2, perspective $\mathrm{C}-$ Implementation process shows the highest positive value of the relation indicator $r_{i}-c_{i}$. It means that this perspective has the most causative impact on other perspectives. 


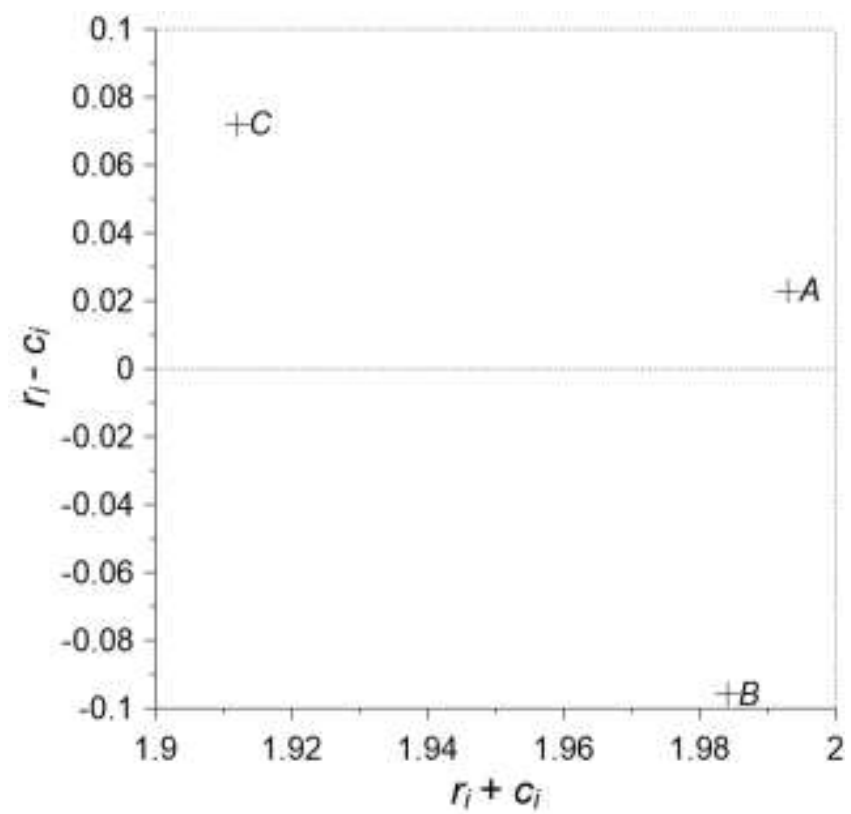

Fig. 2 Influential relation diagram for three perspectives criteria in blockchain supplier selection process

In Figure 3 the influential relation diagram for twelve chosen factors in blockchain integrator selection is presented.

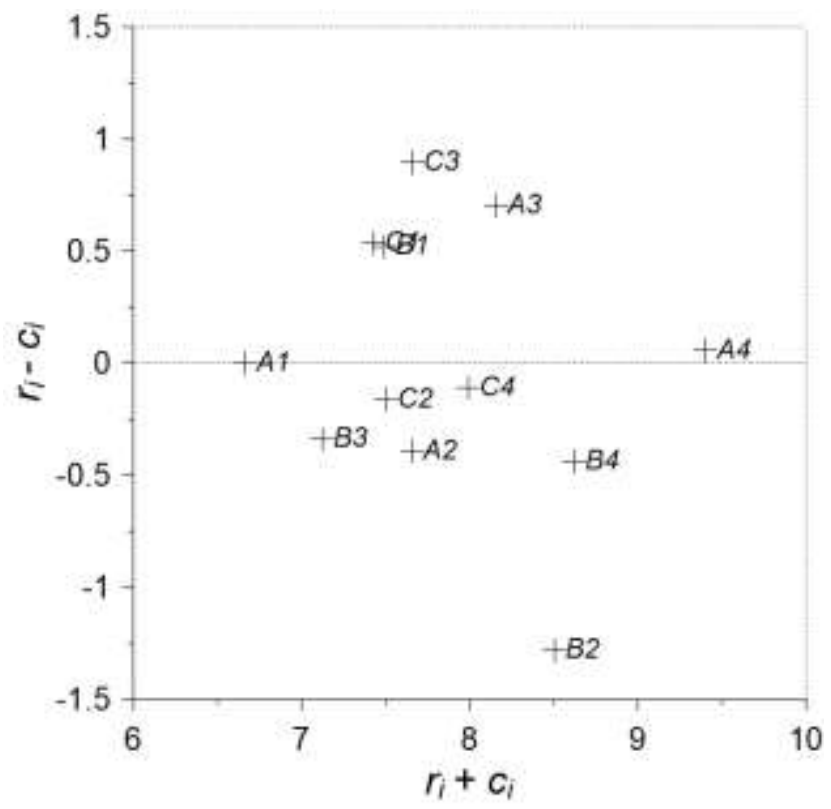

Fig. 3 Influential relation diagram for twelve criteria in blockchain supplier selection process

In Fig. 3, the highest value of the position indicator $r_{i}+c_{i}$ has A4 - Flexibility, from perspective $A$ - Blockchain implementation integrator. It takes up the privileged important position in comparison with others and it is the most crucial criterion in the process of choosing of blockchain integrator. On the other hand, the criterion A1 - Technical capability has the lowest value of this indicator. When it comes to the relation indicator $r_{i}-c_{i}$, the criterion C3 - Roadmap of software development, from the perspective $\mathrm{C}$ - Choice and adaptation of the 
system, with the highest positive value of this indicator, has the strongest impact on the other criteria. Simultaneously, the criterion B2 - Blockchain architecture, with the highest negative value of relation indicator, is the biggest receiver of the impact of the others.

\section{CONCLUSION}

The state-of-the-art Industry 4.0 concept is currently changing the manufacturing processes. To achieve the principles connected with this idea, enterprises need to use massive data transfer securely. One of the solutions from this area is blockchain technology. The article focuses on the analysis of a set of parameters for the assessment and selection of blockchain integrator that can combine blockchain technology with in-house IT systems to support Industry 4.0 processes.

In the article, within the framework of three perspectives, twelve specific parameters have been introduced. According to the experts, the proposed linguistic DEMATEL method is highly suitable for the considered application to analyze the cause-effect relationships among indicators and identify factors which have fundamental importance for blockchain integrator selection.

The reciprocal impact matrices for all pairs of coefficients were specified and the direct-indirect influence matrix of total relations $\boldsymbol{T}$ was obtained, which was used to plot the corresponding diagrams.

According to DEMATEL, the relation and position indicators were received for three perspectives as well as for twelve parameters. The most substantial perspectives and factors have been established. They should be considered as guidelines during the process of choosing a blockchain integrator.

\section{REFERENCES}

Aheleroff, S., Xu, X., Lu, Y., Aristizabal, M. and Valencia, Y. (2020). loT-enabled smart appliances under industry 4.0: A case study. Advanced Engineering Informatics, 43, 101043.

Bai, C., Dallasega, P., Orzes, G., and Sarkis, J. (2020). Industry 4.0 technologies assessment: A sustainability perspective. International Journal of Production Economics, 229, 107776.

Helo, P. and Hao, Y. (2019). Blockchains in operations and supply chains: A model and reference implementation. Computers \& Industrial Engineering, 136, pp. 242-251.

Corallo, A., Lazoi, M. and Lezzi, M. (2020). Cybersecurity in the context of industry 4.0: A structured classification of critical assets and business impacts. Computers in Industry, 114, 103165.

Du, Y.-W. and Zhou, W. (2019). New improved DEMATEL method based on both subjective experience and objective data. Engineering Applications of Artificial Intelligence, 83, pp. 57-71.

Frizzo-Barker, J., Chow-White, P.A., Adams, P.R., Mentanko, J., Ha, H. and Green, S. (2020). Blockchain as a disruptive technology for business: A systematic review. International Journal of Information Management, 51, 102029.

Gabus, A. and Fontela, E. (1972). World problems, an invitation to further thought within the framework of DEMATEL. Geneva: Battelle Geneva Research Center. 
Koh, L., Dolgui, A. and Sarkis, J. (2020). Blockchain in transport and logistics paradigms and transitions. International Journal of Production Research, 58(7), pp. 2054-2062.

Lee, J., Azamfar, M. and Singh, J. (2019). A blockchain enabled Cyber-Physical System architecture for Industry 4.0 manufacturing systems. Manufacturing Letters, 20, pp. 34-39.

Liu, X.L., Wang, W.M., Guo, H., Barenji, A.V., Li, Z. and Huang, G.G. (2020). Industrial blockchain based framework for product lifecycle management in industry 4.0. Robotics and Computer-Integrated Manufacturing, 63(1), 101897.

Reyna, A., Martín, C., Chen, J., Soler, E. and Díaz, M. (2018). On blockchain and its integration with IoT. Challenges and opportunities. Future Generation Computer Systems, 88, pp. 173-190.

Wang, B., Sun, J., He, Y., Pang, D. and Lu, N. (2018). Large-scale Election Based On Blockchain. Procedia Computer Science, 129, pp. 234-237.

Yadav, S., and Singh, S.P. (2020). Blockchain critical success factors for sustainable supply chain. Resources, Conservation and Recycling, 152, 104505.

\begin{abstract}
The article presents the issues covering the modern methods of securing data in both manufacturing processes and companies within the concept of Industry 4.0. In this approach, research problems arose how to implement the right method of secure data sending in sales, manufacturing and distribution processes. It is a very important issue for manufacturing companies as well as how the process of sending electronic data should be safely conducted. While current researches concentrated on the method of blockchain secured electronic documents, there is almost no research concentrating on blockchain integrator selection criteria. The main purpose of this paper is to provide a decision assistance model based on multiple criteria decision analysis technique. Also, mutual relationships between parameters for the assessment of integrators are established. In the article, a Multi-Criteria Decision Analysis (MCDA) was used to assess these characteristics. The Decision Making Trial and Evaluation Laboratory (DEMATEL) technique was chosen for this assessment.
\end{abstract}

Keywords: Blockchain technology, digital signature, data security, Industry 4.0, MCDA 